Quim. Nova, Vol. 36, No. 9, 1388-1390, 2013

\title{
ESTUDIO HIDROFÓBICO DE SOLUCIONES ACUOSAS DILUIDAS DE ISÓMEROS DE PENTANODIOL A VARIAS TEMPERATURAS A PARTIR DE DATOS VOLUMÉTRICOS
}

\author{
Alfonso A. Portacio* \\ Departamento de Matemática y Física, Facultad de Educación y Ciencias, Universidad de Sucre, Cra. 28 \#5-267, Sincelejo - Sucre, \\ Colombia \\ Manuel S. Páez y María del Pilar Urango \\ Departamento de Química, Facultad de Ciencias Básicas, Universidad de Córdoba, Carrera 6 No. 76-103, Km 3, vía Cereté, \\ Córdoba, Colombia \\ Recebido em 16/5/13; aceito em 8/7/13; publicado na web em 9/8/13

\begin{abstract}
HYDROPHOBIC STUDY OF DILUTE AQUEOUS SOLUTIONS OF PENTANEDIOL ISOMERS AT VARIOUS TEMPERATURES FROM VOLUMETRIC DATA. Density of dilute aqueous solutions of 1,2 pentanediol, 2,4 pentanediol, 1,4 pentanediol and 1,5 pentanediol at $283.15,288.15,293.15,298.15,303.15$ and $308.15 \mathrm{~K}$ in the concentration range 0.0000 to 0.0060 in mole fraction were determined by using Wood-Brusie type capillary neck pycnometers. The solute partial molar volume as a function of solute concentration for each system was correlated with a linear equation for each temperature to estimate the slope limit and the partial molar volume at infinite dilution, and the predominant hydrophilic-hydrophobic effect was found in this region.
\end{abstract}

Keywords: pentanediol; hydrophobicity; density.

\section{INTRODUCCIÓN}

Las moléculas anfifílicas son un tipo de compuestos formados por un fragmento hidrofóbico y otro hidrofílico que poseen una fuerte tendencia a formar ensamblajes altamente organizados debido a la participación de diferentes tipos de interacciones no-covalentes, principalmente enlaces de hidrógeno y fuerzas de van der Waals. ${ }^{1}$ Por otra parte, las soluciones acuosas de alcoholes han sido extensamente estudiadas en busca de una explicación del comportamiento que estas presentan particularmente a baja concentración y que se ha atribuido a la solvatación hidrofóbica. ${ }^{2}$ Por tal motivo, se han desarrollado múltiples estudios sobre el comportamiento de las soluciones acuosas de solutos apolares y mixtos, para tratar de entender el predominio del efecto hidrofóbico o hidrofílico dependiendo de la concentración de soluto, con lo cual los dioles en virtud de su carácter mixto se constituyen como candidatos naturales (modelos) que ayudarían a entender dicho predominio en las interacciones de las biomoleculas en un ambiente acuoso. ${ }^{3-8}$ También la influencia de la temperatura sobre las propiedades volumétricas de soluciones acuosas ha sido frecuentemente usada para obtener información sobre los efectos de distintos solutos anfifílicos sobre la estructura del agua, a partir de datos de volúmenes molares parciales a dilución infinita a fin de determinar el predominio hidrofóbico o hidrofílico de los solutos y su efecto sobre la estructura del agua. ${ }^{8,9}$

\section{PARTE EXPERIMENTAL}

Como reactivos se utilizaron (1,2 pentanodiol, 2,4 pentanodiol, 1,4 pentanodiol y 1,5 pentanodiol) de $99 \%$ de pureza, Aldrich. El agua empleada para la calibración de los equipos y preparación de las soluciones fue doblemente destilada, con una conductividad menor que $2 \mu \mathrm{S} \mathrm{cm}^{-1}$. Todas las soluciones fueron preparadas por el método de pesada usando una balanza OHAUS con una sensibilidad de $\pm 10^{-5} \mathrm{~g}$ en el rango más bajo. La densidad de las soluciones fue medida usando picnómetros de cuello capilar, tipo Wood-Brusie ${ }^{10}$ con

*e-mail: alfonsoportacio@gmail.com una incertidumbre de $\pm 10^{-5} \mathrm{~g} \mathrm{~cm}^{-3}$. La capacidad de estos picnómetros es de aproximadamente $80 \mathrm{~cm}^{3}$, cuales fueron calibrados con agua a las temperaturas de trabajo. Todas las medidas fueron realizadas en baños a temperatura constante y sus variaciones fueron monitoreadas con un termómetro digital FlukeHartScientific con una precisión de $\pm 0.001^{\circ} \mathrm{C}$.

\section{RESULTADOS Y DISCUSIÓN}

Los resultados obtenidos experimentalmente para las densidades ( $\rho$ ) de las soluciones acuosas de 1,2 pentanodiol, 2,4 pentanodiol, 1,4 pentanodiol y 1,5 pentanodiol a $(283,15 ; 288,15 ; 293,15 ; 298,15$; $303,15$ y 308,15$) \mathrm{K}$, en el intervalo de concentración entre $(0.0000$ 0.0060) en fracción molar aparecen reportados en la Tabla 1, el volumen molar parcial del soluto se calcula a partir de la ecuación (1). Estos datos están buen acuerdo, en tendencias y en orden de magnitud con otros trabajos similares..$^{11,12}$

$$
\bar{V}_{2}=V_{m}+x_{1}\left(\partial V_{m} / \partial x_{2}\right)_{T, P}
$$

Donde $V_{m}$ es el volumen molar, $\bar{V}_{2}$ es el volumen molar parcial del soluto, $x_{1}$ y $x_{2}$ son las fracciones molares del solvente y el soluto. Estudios similares muestran que, para los dioles el volumen molar parcial del soluto en la región diluida tiene un comportamiento lineal con la concentración de soluto para cada temperatura, esta correlación viene dada por la siguiente ecuación: ${ }^{9}$

$$
\bar{V}_{2}=x_{2}\left(\partial \bar{V}_{2} / \partial x_{2}\right)_{x_{2} \rightarrow 0}+\bar{V}_{2}^{\infty}
$$

Donde $\left(\partial \bar{V}_{2} / \partial x_{2}\right)_{x_{2} \rightarrow 0}$ y $\bar{V}_{2}^{\infty}$ son la pendiente límite y el volumen molar parcial a dilución infinita respectivamente, los resultados de estas cantidades para las soluciones acuosas diluidas de 1,2 pentanodiol, 2,4 pentanodiol, 1,4 pentanodiol y 1,5 pentanodiol se muestran para cada temperatura en las Tabla 2 y Tabla 3 respectivamente.

El análisis de los resultados de la Tabla 2, indica el predominio de 
la interacción hidrofóbica sobre la hidrofílica, además también muestra la disminución de este dominio con el aumento de la temperatura para todo los solutos, en virtud a que la pendiente límite es negativa y que esta se hace más positiva con el aumento de la temperatura. ${ }^{9} \mathrm{El}$ carácter hidrofóbico disminuye en el orden 1,2 PD >1,5PD >1,4PD>2,4 PD por el hecho de que en este orden la pendiente límite se hace más positiva, indicándose de esta forma que la posición de los grupos

Tabla 1. Densidades $(\rho)$ de las soluciones acuosas de 1,2 pentanodiol, 2,4 pentanodiol, 1,4 pentanodiol y 1,4 pentanodiol a $(283.15 ; 288.15 ; 293.15$; $298.15 ; 303.15$ y 308.15$) \mathrm{K}$

\begin{tabular}{|c|c|c|c|c|c|c|c|}
\hline \multirow{12}{*}{ 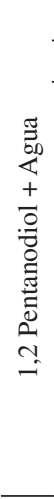 } & $\mathrm{T}[\mathrm{K}]$ & 283.15 & 288.15 & 293.15 & 298.15 & 303.15 & 308.15 \\
\hline & $\mathrm{x}_{2}$ & \multicolumn{6}{|c|}{$\rho\left[\mathrm{g} \mathrm{cm}^{-3}\right]$} \\
\hline & 0.0000 & 0.99970 & 0.99910 & 0.99821 & 0.99705 & 0.99565 & 0.99403 \\
\hline & 0.0020 & 0.99995 & 0.99933 & 0.99844 & 0.99728 & 0.99586 & 0.99425 \\
\hline & 0.0025 & 1.00002 & 0.99939 & 0.99850 & 0.99733 & 0.99592 & 0.99431 \\
\hline & 0.0030 & 1.00010 & 0.99946 & 0.99856 & 0.99740 & 0.99598 & 0.99437 \\
\hline & 0.0035 & 1.00018 & 0.99953 & 0.99863 & 0.99746 & 0.99604 & 0.99443 \\
\hline & 0.0040 & 1.00026 & 0.99961 & 0.99871 & 0.99753 & 0.99610 & 0.99449 \\
\hline & 0.0045 & 1.00035 & 0.99968 & 0.99878 & 0.99760 & 0.99616 & 0.99456 \\
\hline & 0.0050 & 1.00044 & 0.99976 & 0.99886 & 0.99768 & 0.99622 & 0.99463 \\
\hline & 0.0055 & 1.00054 & 0.99985 & 0.99894 & 0.99775 & 0.99628 & 0.99469 \\
\hline & 0.0060 & 1.00064 & 0.99993 & 0.99903 & 0.99783 & 0.99635 & 0.99476 \\
\hline \multirow{12}{*}{ 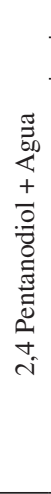 } & $\mathrm{T}[\mathrm{K}]$ & 3.15 & 288.15 & 93.15 & 298.15 & 303.15 & 308.15 \\
\hline & $\mathrm{x}_{2}$ & \multicolumn{6}{|c|}{$\rho\left[\mathrm{g} \mathrm{cm}^{-3}\right]$} \\
\hline & 0.0000 & 0.99970 & 0.99910 & 0.99821 & 0.99705 & 0.99565 & 0.99403 \\
\hline & 0.0020 & 0.99973 & 0.99911 & 0.99821 & 0.99706 & 0.99566 & 0.99405 \\
\hline & 0.0025 & 0.99974 & 0.99911 & 822 & 0.99706 & 0.99 & 0.99405 \\
\hline & 0.0030 & 0.99975 & 0.99912 & 0.99822 & 0.99706 & 0.99567 & 0.99406 \\
\hline & 0.0035 & 0.99976 & 0.99912 & 0.99823 & 0.99707 & 0.99567 & 0.99407 \\
\hline & 0.0040 & 0.99978 & 0.99913 & 0.99823 & 0.99708 & 0.99568 & 0.99408 \\
\hline & 0.0045 & 0.99980 & 0.99914 & 0.99824 & 0.99708 & 0.99569 & 0.99408 \\
\hline & 0.0050 & 0.99982 & 0.99915 & 0.99825 & 0.99709 & 0.99569 & 0.99409 \\
\hline & 0.0055 & 0.99984 & 0.99916 & 0.99826 & 0.99710 & 0.99570 & 0.99410 \\
\hline & 0.0060 & 0.99987 & 0.99917 & 0.99827 & 0.99711 & 0.99571 & 0.99411 \\
\hline \multirow{12}{*}{ 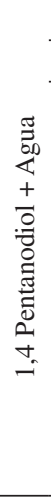 } & $\mathrm{T}[\mathrm{K}]$ & 15 & 5 & 3.15 & 298.15 & 5 & 5 \\
\hline & $\mathrm{x}_{2}$ & \multicolumn{6}{|c|}{$\rho\left[\mathrm{g} \mathrm{cm}^{-3}\right]$} \\
\hline & 0.0000 & 0.99970 & 0.99910 & 0.99821 & 0.99705 & 0.99565 & 0.99403 \\
\hline & 0.0020 & 0.99963 & 0.99904 & 0.99814 & 0.99699 & 0.99558 & 0.99397 \\
\hline & 0.0025 & 0.99962 & 0.99902 & 0.99812 & 0.99699 & 0.99556 & 0.99396 \\
\hline & 0.0030 & 0.99961 & 0.99902 & 0.99812 & 0.99699 & 0.99556 & 0.99396 \\
\hline & 0.0035 & 0.99961 & 0.99902 & 0.99812 & 0.99700 & 0.99555 & 0.99397 \\
\hline & 0.0040 & 0.99962 & 0.99903 & 0.99813 & 0.99701 & 0.99556 & 0.99398 \\
\hline & 0.0045 & 0.99963 & 0.99905 & 0.99814 & 0.99703 & 0.99557 & 0.99399 \\
\hline & 0.0050 & 0.99964 & 0.99906 & 0.99815 & 0.99705 & 0.99558 & 0.99401 \\
\hline & 0.0055 & 0.99966 & 0.99908 & 0.99817 & 0.99708 & 0.99559 & 0.99403 \\
\hline & 0.0060 & 0.99968 & 0.99911 & 0.99819 & 0.99710 & 0.99561 & 0.99404 \\
\hline \multirow{12}{*}{ 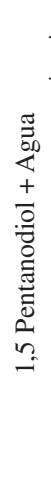 } & $\mathrm{T}[\mathrm{K}]$ & 283.15 & 288.15 & 293.15 & 298.15 & 303.15 & 308.15 \\
\hline & $\mathrm{x}_{2}$ & \multicolumn{6}{|c|}{$\rho\left[\mathrm{g} \mathrm{cm}^{-3}\right]$} \\
\hline & 0.0000 & 0.99970 & 0.99910 & 0.99821 & 0.99705 & 0.99565 & 0.99403 \\
\hline & 0.0020 & 0.99970 & 0.99911 & 0.99821 & 0.99705 & 0.99563 & 0.99402 \\
\hline & 0.0025 & 0.99970 & 0.99911 & 0.99821 & 0.99709 & 0.99563 & 0.99402 \\
\hline & 0.0030 & 0.99971 & 0.99913 & 0.99822 & 0.99710 & 0.99563 & 0.99402 \\
\hline & 0.0035 & 0.99973 & 0.99914 & 0.99824 & 0.99705 & 0.99564 & 0.99402 \\
\hline & 0.0040 & 0.99975 & 0.99917 & 0.99826 & 0.99706 & 0.99564 & 0.99402 \\
\hline & 0.0045 & 0.99977 & 0.99919 & 0.99828 & 0.99707 & 0.99565 & 0.99402 \\
\hline & 0.0050 & 0.99980 & 0.99922 & 0.99831 & 0.99708 & 0.99566 & 0.99403 \\
\hline & 0.0055 & 0.99983 & 0.99925 & 0.99833 & 0.99710 & 0.99566 & 0.99403 \\
\hline & 0.0060 & 0.99987 & 0.99929 & 0.99836 & 0.99712 & 0.99568 & 0.99404 \\
\hline
\end{tabular}

Tabla 2. Pendientes límites para soluciones acuosas de 1,2 Pentanodiol, 2,4 Pentanodiol, 1,4 Pentanodiol y 1,5 Pentanodiol a $(283.15 ; 288.15 ; 293.15$; $298.15 ; 303.15$ y 308.15$) \mathrm{K}$

\begin{tabular}{ccccc}
\hline & $1,2 \mathrm{PD}$ & $1,5 \mathrm{PD}$ & $1,4 \mathrm{PD}$ & $2,4 \mathrm{PD}$ \\
\hline $\mathrm{T}[\mathrm{K}]$ & & $\left(\partial \bar{V}_{2} / \partial x_{2}\right)_{x_{2} \rightarrow 0}\left[\mathrm{~cm}^{3} / \mathrm{mol}\right]$ & \\
\hline 283.15 & -440.7 & -394.39 & -188.0 & -167.9 \\
288.15 & -329.2 & -384.14 & -182.0 & -94.5 \\
293.15 & -297.9 & -341.29 & -172.0 & -76.8 \\
298.15 & -248.0 & -286.12 & -170.0 & -75.4 \\
303.15 & -150.3 & -163.58 & -164.0 & -55.7 \\
308.15 & -127.3 & -112.82 & -118.0 & -53.9 \\
\hline
\end{tabular}

Tabla 3. Volumen molar parcial a dilución infinita para soluciones acuosas de 1,2 Pentanodiol, 2,4 Pentanodiol, 1,4 Pentanodiol y 1,5 Pentanodiol a (283.15; $288.15 ; 293.15 ; 298.15 ; 303.15$ y 308.15$) \mathrm{K}$

\begin{tabular}{|c|c|c|c|c|}
\hline & $1,5 \mathrm{PD}$ & 1,4 PD & $2,4 \mathrm{PD}$ & $1,2 \mathrm{PD}$ \\
\hline $\mathrm{T}[\mathrm{K}]$ & \multicolumn{4}{|c|}{$\bar{V}_{2}^{\infty}\left[\mathrm{cm}^{3} / \mathrm{mol}\right]$} \\
\hline 283.15 & 105.30 & 104.61 & 104.60 & 103.10 \\
\hline 288.15 & 105.32 & 104.76 & 104.69 & 103.13 \\
\hline 293.15 & 105.35 & 104.92 & 104.77 & 103.17 \\
\hline 298.15 & 105.38 & 105.10 & 104.87 & 103.22 \\
\hline 303.15 & 105.43 & 105.26 & 104.97 & 103.29 \\
\hline 308.15 & 105.48 & 105.44 & 105.07 & 103.39 \\
\hline
\end{tabular}

Tabla 4. Constantes de ajuste para la dependencia cuadrática del $\bar{V}_{2}^{\infty}$ con temperatura para soluciones acuosas diluidas de 1,2 pentanodiol, 2,4 pentanodiol, 1,4 pentanodiol y 1,5 pentanodiol

\begin{tabular}{ccccccc}
\hline & $\mathrm{c}_{1}$ & $\sigma \mathrm{c}_{1}$ & $\mathrm{c}_{2}$ & $\sigma \mathrm{c}_{2}$ & $\mathrm{c}_{3}$ & $\sigma \mathrm{c}_{3}$ \\
\hline $1,2 \mathrm{PD}$ & 0.00034 & 0.00004 & -0.19 & 0.02 & 129 & 3 \\
$1,5 \mathrm{PD}$ & 0.00016 & 0.00002 & -0.19 & 0.01 & 118 & 2 \\
$1,4 \mathrm{PD}$ & 0.00011 & 0.00004 & -0.133 & 0.002 & 105 & 3 \\
$2,4 \mathrm{PD}$ & 0.00009 & 0.00003 & -0.14 & 0.02 & 107 & 2 \\
\hline
\end{tabular}

hidroxilos se constituye en un factor importante a la hora de realizar este tipo de análisis. Es evidente que el 1,2 pentanodiol presenta la mayor parte alquílica expuesta al solvente, esta podría ser la razón por la cual presentaría el mayor carácter hidrofóbico. En tanto que el 1,5 pentanodiol también presenta una buena parte expuesta al solvente pero en una posición intermedia.

Por otra parte, es conocido que la segunda derivada del volumen molar parcial a dilución infinita con respecto a la temperatura, refleja el efecto de los solutos sobre la estructura del agua e indica el comportamiento hidrofóbico del soluto. El signo positivo se atribuye a que el soluto tiene un efecto formador sobre la estructura del agua y el signo negativo a que el soluto tiene un efecto disruptor. ${ }^{13}$ Siguiendo este orden de ideas un ajuste funcional del $\bar{V}_{2}^{\infty}$ con la temperatura mediante la ecuación cuadrática es presentado en la ecuación (3) y sus constantes de ajuste en la Tabla 4; de acuerdo con la interpretación antes dada, podemos decir que en la región diluida los isómeros de pentanodiol ejercen un efecto formador sobre la estructura del agua, ratificando de esta forma lo obtenido con la pendiente límite, por cuantola segunda derivada del volumen molar parcial a dilución infinita con respecto a la temperatura es positiva, además esta se hace 
más pequeña en el orden 1,2 PD> 1,5PD > 1,4PD > 2,4PD, reflejando así que este efecto cambia con la posición de los grupos hidroxilos.

$$
\bar{V}_{2}^{\infty}=c_{1} T^{2}+c_{2} T+c_{3}
$$

\section{CONCLUSIONES}

Se reportan datos experimentales para la densidad en función de la concentración en la región diluida en el intervalo $(0.0000-0.0060)$ en fracción molar a las temperaturas entre $(283.15$ - 308.15) K. para los sistemas 1,2 pentanodiol, 2,4 pentanodiol, 1,4 pentanodiol y 1,5 pentanodiol. También se reportan datos de volúmenes molares parciales a dilución infinita para el soluto a las temperaturas señaladas y los sistemas estudiados.

Los resultados obtenidos con los criterios, pendiente límite del volumen molar parcial y segunda derivada del volumen molar parcial a dilución infinita con respecto a la temperatura, muestran que en la región diluida los isómeros de pentanodiol tienen un efecto formador sobre la estructura del agua, debido predominio del efecto hidrofóbico sobre el hidrofílico. Además este predominio disminuye con el aumento de la temperatura y con la posición de los grupos hidroxilos en el orden 1,2 PD>1,5PD>1,4PD>2,4 PD.

\section{REFERENCIAS}

1. Fernández, G.; García, F.; Aparicio, F.; Sánchez, L.; An. Quim. 2009, $105,89$.

2. Jiménez, E.; Romero, C.; Suárez, F.; J. Chem. Thermodyn. 2009, 41, 513.

3. Romero, C.; Páez, M.; J. Therm. Anal. Calorim. 2002, 70, 263.

4. Romero, C; Páez, M.; Arteaga, J.; Romero, M.; Negrete, F.; J. Chem. Thermodyn. 2007, 39. 1101.

5. Bushuev, Y.; Davletbaeva, S.; Korolev, V.; Russ. Chem. Bull. 2008, 57, 1811.

6. Páez, M.; Lafont, J.; Portacio, A.; Información Tecnológica 2009, 20, 55 .

7. Portacio, A.; Páez, M.; Ramírez, D.; Espitia, I.; García, J; Rev. Col. Fís. 2009, 41, 378.

8. Páez, M.; Romero, C.; J. Solution Chem. 2007, 36, 237.

9. Páez, M.; Portacio, A.; Ortega, C.; Información Tecnológica 2011, 22, 59.

10. Wood, S.; Brusie, J.; J. Am. Chem. Soc. 1943, 65, 1891.

11. Kapadi, U.; Hundiwale, D.; Patil, N.; Lande, M.; Patil, P.; Fluid Phase Equilib. 2001, 192, 63.

12. George, J.; Sastry, N.; J. Chem. Eng. Data 2003, 48, 1529.

13. Banipal, P.; Chahal, A.; Banipal, T.; J. Chem. Thermodyn. 2009, 41, 452. 